\title{
BMJ Open PrEdiction of Risk and Communication of outcomE followIng major lower limb amputation: a collaboratiVE study (PERCEIVE) - protocol for the PERCEIVE qualitative study
}

\author{
Sarah Milosevic (D) , ${ }^{1}$ Lucy Brookes-Howell, ${ }^{1}$ Brenig Llwyd Gwilym (D) , \\ Cherry-Ann Waldron (D) , ${ }^{1}$ Emma Thomas-Jones, ${ }^{1}$ Ryan Preece, ${ }^{3}$ \\ Philip Pallmann (D) , ${ }^{1}$ Debbie Harris, ${ }^{1}$ Ian Massey, ${ }^{4}$ Philippa Stewart, ${ }^{5}$ \\ Katie Samuel, ${ }^{6}$ Sian Jones, ${ }^{7}$ David Cox,${ }^{7}$ Christopher P Twine, ${ }^{8}$ \\ Adrian Edwards (i) , ${ }^{9}$ David C Bosanquet ${ }^{2}$
}

To cite: Milosevic S, BrookesHowell L, Gwilym BL, et al. PrEdiction of Risk and Communication of outcomE following major lower limb amputation: a collaboratiVE study (PERCEIVE) protocol for the PERCEIVE qualitative study. BMJ Open 2022;12:e053159. doi:10.1136/ bmjopen-2021-053159

- Prepublication history and additional supplemental material for this paper are available online. To view these files, please visit the journal online (http://dx.doi.org/10.1136/ bmjopen-2021-053159).

Received 05 May 2021 Accepted 03 December 2021

Check for updates

(C) Author(s) (or their employer(s)) 2022. Re-use permitted under CC BY-NC. No commercial re-use. See rights and permissions. Published by BMJ.

For numbered affiliations see end of article.

Correspondence to

Dr Sarah Milosevic;

milosevics@cardiff.ac.uk

\section{ABSTRACT}

Introduction Deciding whether to proceed with a major lower limb amputation is life-changing and complex, and it is crucial that the right decision is made at the right time. However, medical specialists are known to poorly predict risk when assessing patients for major surgery, and there is little guidance and research regarding decisions about amputation. The process of shared decision-making between doctors and patients during surgical consultations is also little understood. Therefore, the aim of this study is to analyse in depth the communication, consent, risk prediction and decision-making process in relation to major lower limb amputation.

Methods and analysis Consultations between patients and surgeons at which major lower limb amputation is discussed will be audio-recorded for 10-15 patients. Semi-structured follow-up interviews with patients (and relatives/carers) will then be conducted at two time points: as soon as possible/ appropriate after a decision has been reached regarding surgery, and approximately 6 months later. Semi-structured interviews will also be conducted with 10-15 healthcare professionals working in the UK National Health Service (NHS) involved in amputation decision-making. This will include surgeons, anaesthetists and specialist physiotherapists at 2-4 NHS Health Boards/Trusts in Wales and England. Discourse analysis will be used to analyse the recorded consultations; interviews will be analysed thematically. Finally, workshops will be held with patients and healthcare professionals to help synthesise and interpret findings.

Ethics and dissemination The study has been approved by Wales REC 7 (20/WA/0351). Study findings will be published in international peer-reviewed journal(s) and presented at national and international scientific meetings. Findings will also be disseminated to a wide NHS and lay audience via presentations at meetings and written summaries for key stakeholder groups.

\section{INTRODUCTION}

Major lower limb amputation (MLLA) is a life-changing, high-risk procedure, ${ }^{1-4}$
Strengths and limitations of this study

- The study will capture a wide range of perspectives from key stakeholders in amputation decision-making.

- The inclusion of several data sources will enable a rich understanding of the amputation decisionmaking process.

- Recording consultations between patients and surgeons will provide insight into the real-life process of surgical decision-making - an area that is currently little understood.

- Strong patient involvement, from study development through to analysis and dissemination, will enhance the validity and reach of findings.

- As this is an exploratory study, participants will be recruited from a relatively small number of sites.

and deciding whether to proceed with an amputation can be extremely difficult. ${ }^{5}$ For patients with extensive foot wounds and/or pain (due to diabetes or peripheral arterial disease), this decision may involve balancing up the risks and benefits of surgery with non-operative management. If frail patients develop lower limb complications, which can only be resolved by amputation, patients (and relatives/carers) face a difficult decision of surgery versus palliative symptom control. Making the right decision at the right time is crucial. A wrong or mis-timed decision can result in reduced quality of life, patient and/ or family regret, poorer patient outcomes and increased costs. ${ }^{367}$

To inform decisions in relation to MLLA, healthcare professionals estimate the likely risks (including mortality) and benefits (such 
as the chance of mobilisation). However, medical specialists are known to poorly predict risks and outcomes (especially longer term functional outcomes) when assessing patients for major surgery. ${ }^{8}$ In vascular surgery, this could lead to inappropriate amputations or an unnecessary delay to amputation. As there is currently limited evidence relating to the outcomes of amputation, ${ }^{9}$ shared decision-making between patients and clinicians is particularly important and may result in superior outcomes, for example, in relation to quality of life. ${ }^{10} 11$

Shared decision-making is considered fundamental to good medical practice.$^{12}$ However, in surgery, this is in its infancy, and future studies are needed to improve shared decision-making during surgical consultations. ${ }^{13}$ For example, it is not currently known how well MLLA risk information is communicated, and how effective patientsurgeon communication is in enabling decision-making based on what is most important to the patient. ${ }^{14}$

The top research priority identified by healthcare professionals in the James Lind Alliance Priority Setting Partnership for Vascular Surgery was to investigate how outcomes in critical limb ischaemia could be improved, including how best to decide whether to proceed to revascularisation, amputation or palliation. ${ }^{15}$ There are extensive guidelines on when to undertake revascularisation, and by which techniques, based on large, well-conducted randomised controlled trials. ${ }^{16}$ In contrast, guidance and research regarding amputation decision-making is almost non-existent, ${ }^{5}$ leading to variations in clinical decisionmaking. ${ }^{17}$ Key to addressing this research priority is, therefore, to explore amputation decision-making and risk perception in detail.

\section{Study objectives}

The primary objective of this study is to analyse in-depth the communication, consent, risk prediction and decision-making process in relation to MLLA, examined via patient-surgeon consultations and individual patient and healthcare professional interviews. The secondary objectives are to:

- Explore and describe how risks are communicated and options discussed with patients and relatives/ carers, assessing the extent of shared decision-making in patient-surgeon consultations.

- Explore and describe patients' perceptions of decision-making, the communication of risks and benefits of MLLA, expectations of rehabilitation (and whether these are met) and any decisional regret.

- Explore and describe how healthcare professionals evaluate risks and outcomes when considering MLLA.

- Propose (an) intervention(s) to improve shared decision-making and risk perception/communication around MLLA, together with a logic model for the intervention and its future evaluation.

The PERCEIVE study (PrEdiction of Risk and Communication of outcomE followIng MLLA-A collaboratiVE study) also comprises a parallel quantitative component; an international multicentre prospective observational cohort service evaluation, aiming to evaluate the accuracy of outcome predictions made by healthcare professionals and risk prediction tools for patients undergoing MLLA. ${ }^{18}$ Qualitative and quantitative findings will be synthesised in the final stage of the study. This paper presents the protocol for the qualitative and data synthesis components of PERCEIVE.

\section{METHODS AND ANALYSIS \\ Study design}

The PERCEIVE study will commence on 1 October 2020 and the planned end date is 30 September 2022. The qualitative study will be carried out in 2-4 participating NHS Health Boards/Trusts in Wales and England. A Principal Investigator (PI) will be identified at each site; Associate PIs may also be identified to assist with study processes.

This qualitative study will comprise: (1) audio-recording of consultations between patients and surgeons, (2) follow-up interviews with patients and relatives/carers and (3) interviews with healthcare professionals (see online supplemental information 1 file for full interview topic guides). All study interviews will be conducted via telephone or video call, depending on the preference of the participant. See figure 1 for a schematic overview of study processes.

\section{Participant sampling}

In total, 10-15 healthcare professionals will be purposively sampled, with the aim to include 8-10 surgeons, 1-3 anaesthetists and 1-3 specialist physiotherapists from several Health Boards/Trusts. A total of 10-15 patients will be purposively sampled to include variation where possible in gender, age, MLLA status (above knee/below knee/no amputation and first/second amputation) and Health Board/Trust. Participants will be eligible for the study if they meet one of the following inclusion criteria and none of the exclusion criteria apply.

\section{Inclusion criteria}

- Any patient aged 18 years old or over with chronic limb-threatening ischaemia (due to vascular disease) or significant diabetic foot disease for whom MLLA is considered or discussed (and their relatives/carers)

- Vascular surgeons/anaesthetists/specialist physiotherapists involved in, or supporting, MLLA decision-making

\section{Exclusion criteria}

- Patients aged under 18 years old.

- Patients undergoing MLLA for other causes (eg, cancer, trauma).

- Any patient/healthcare professional unable or unwilling to provide informed consent. Some patients undergoing emergency MLLA will have insufficient time to give informed consent.

- Potential participants with an insufficient understanding of English or Welsh to be able to provide informed consent. 
Figure 1: Study data collection

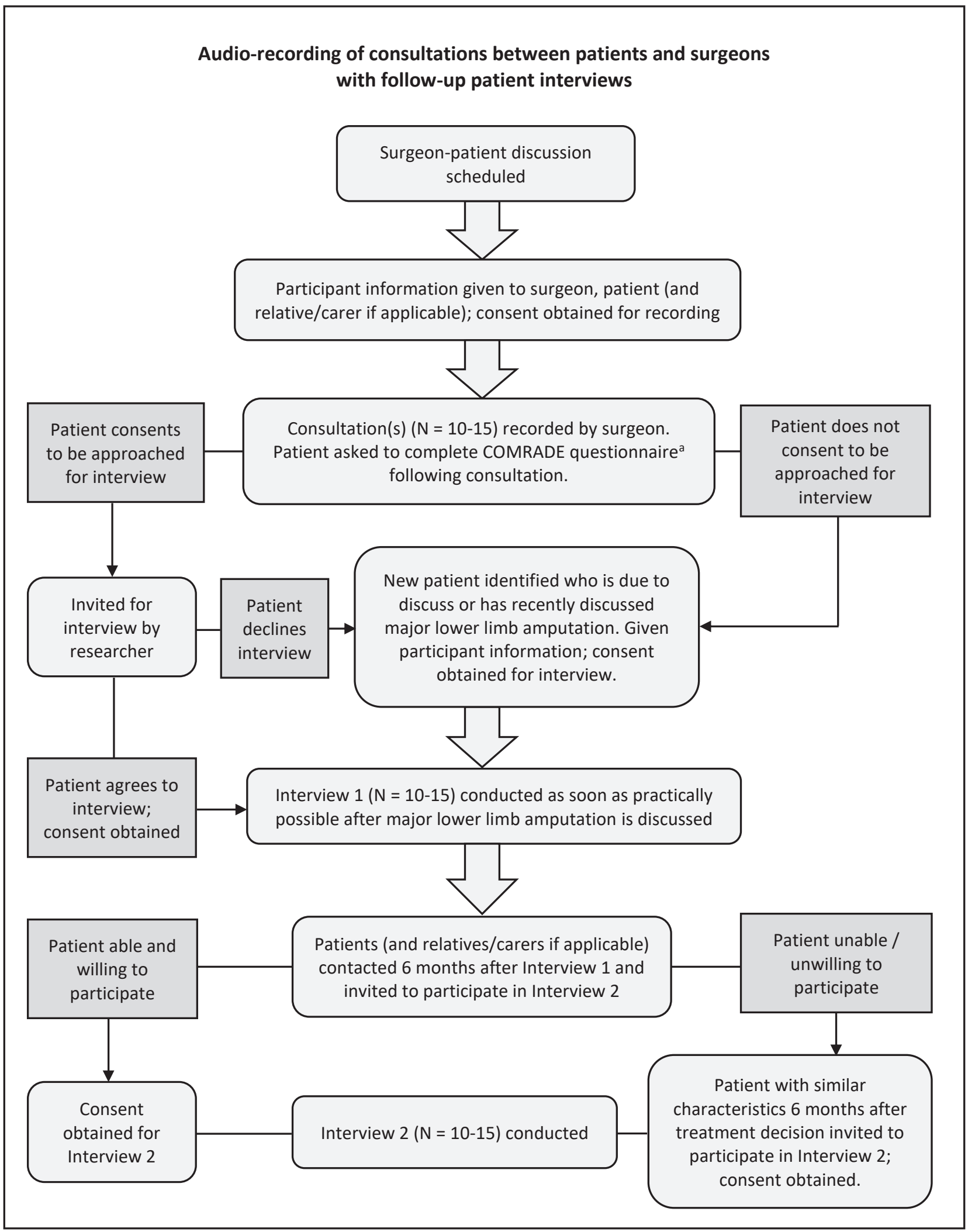

Figure 1 Study data collection.

${ }^{\text {a }}$ COMRADE questionnaire: A patient-based outcome measure for risk communication and treatment decision making effectiveness. 
- Potential participants unable to complete an interview in English.

\section{Sample size}

The study will include $10-15$ patients interviewed at two time points and 10-15 healthcare professional interviews (resulting in 30-45 interviews), together with 10-15 patient-surgeon consultations (potentially with multiple consultation recordings). It is anticipated that this sample size will enable us to achieve sufficient information power, ${ }^{19}$ as the study is exploratory, the participant groups and study aims are highly specific, and in-depth analysis will be conducted. The research team will make pragmatic decisions on sample size in accordance with ongoing appraisal of information power and purposive sampling requirements.

\section{Methods of data collection}

Audio-recording of consultations between patients and surgeons

Surgeons will be asked to audio-record consultations with patients (and relatives/carers) at which MLLA is discussed, to identify how risks are communicated and options discussed during decision-making. As informed consent for surgery is an ongoing process, ${ }^{20}$ several relevant surgeon-patient consultations may be audiorecorded for each patient. As soon as possible after each consultation, patients will be asked to complete a COMRADE (combined outcome measure for risk communication and treatment decision making effectiveness) questionnaire, ${ }^{21}$ a patient-based outcome measure evaluating the effectiveness of risk communication and decision-making satisfaction in consultations. The extent of shared decision-making in the recorded consultation (or series of consultations) will be assessed by a qualitative researcher, using the Observer OPTION V.5 Item measure. ${ }^{22}$ Where possible, scores from these tools will be used to focus discussions in the patient and surgeon follow-up interviews.

\section{Follow-up interviews with patients and relatives/carers}

Semi-structured interviews will be conducted with patients (and relatives/carers) as soon as possible/appropriate after a decision has been reached regarding MLLA surgery (including some where the decision is not to have an amputation), with timing being guided by the clinical team. If possible, all interviews will be conducted with patients recruited for the audio-recorded consultations. Interviews will explore patients' (and relatives'/ carers') perceptions of the risks and benefits of MLLA, influences on their decision (eg, current health/pain/ mobility status) and rehabilitation expectations. Interviewees will also be asked about their satisfaction with the consultation discussion(s), feelings about the timing of the decision-making, and preferences regarding how risks and benefits/outcomes are communicated to them by healthcare professionals.

Semi-structured follow-up interviews will be conducted with patients (and relatives/carers), approximately 6
$( \pm 2)$ months after their initial interviews. Interviews will explore any decisional regret, whether expectations have been met, and with the benefit of hindsight, if patients would have preferred anything to be done differently regarding MLLA communication and the decisionmaking and consent process (including relating to the timing of their decision).

\section{Interviews with healthcare professionals}

Semi-structured interviews will be conducted with surgeons, anaesthetists and specialist physiotherapists. This mix will capture views from all those involved in supporting and overseeing MLLA decision-making. Interviews will explore how healthcare professionals evaluate risks and outcomes when discussing MLLA and identify clinical and non-clinical factors that influence decisionmaking. Interviewees will also be asked about their approaches to communicating with patients and their families and their approaches to shared decision-making.

\section{Participant recruitment}

Audio-recording of consultations between patients and surgeons

The site PI, Associate PI or research nurse will identify potential surgeons for participation in the study and provide them with a participant information sheet, which will include information about the audio-recording of consultation(s) and the healthcare professional interviews. Surgeons who agree for their consultation(s) to be recorded will be asked to complete a consent form.

Participating surgeons will be asked to identify patients due to discuss the possibility of MLLA, in accordance with the study inclusion/exclusion criteria and purposive sampling requirements. Patients who are considered potentially suitable for inclusion (and any relatives/carers due to be present at the consultation) will be provided with a participant information sheet by the site PI, Associate PI or research nurse or a member of the clinical team. If consultations are taking place remotely (eg, via video or telephone call), the participant information may be read out to patients over the telephone. The participant information sheet will include information about the audio-recording of consultation(s) and the patient interviews. If patients agree for their consultation(s) to be recorded, they (and any relatives/carers) present at the consultation(s) will be asked to give verbal consent before the start of the consultation. Consent will be taken by the research nurse or a GCP (Good Clinical Practice) - trained member of the healthcare team delegated by the PI and will be audio-recorded. As part of the consent process, patients/relatives/carers will be asked whether they are willing to be approached by a researcher to take part in an interview shortly after the consultation(s).

Follow-up interviews with patients and relatives/carers

Where possible, interviews will be conducted with patients (and relatives/carers) who have taken part in an audiorecorded consultation and consented to be approached for an interview. If there is a need to recruit patients 
who have not previously taken part in an audio-recorded consultation, a member of the clinical team will identify patients who have recently discussed the possibility of MLLA, in accordance with the study inclusion/exclusion criteria. Patients will be invited to take part in an interview as soon as possible/appropriate after a decision has been reached regarding MLLA surgery, with timing being guided by the clinical team. Patients will be asked during the consent process whether they would be willing to be approached for a second interview, approximately 6 months after the initial interview.

Patients who indicate they are willing to be approached for a second interview will be contacted by the qualitative researcher approximately 6 months $( \pm 2$ months) after the initial interview. Where patients cannot be contacted directly, the qualitative researcher will attempt to contact them via the clinical team or using contact details of a close friend or family member provided by the patient. If patients have not consented to be contacted for a second interview, cannot be contacted, or are unable or unwilling to take part at the time of invitation, a member of the clinical team will identify alternative patients who are at the 6 -month ( \pm 2 months) point following a decision about MLLA, closely matched to the original participants in terms of gender, age and MLLA status.

\section{Interviews with healthcare professionals}

The site PI, Associate PI or research nurse will identify potential healthcare professionals for participation in the study. All surgeons who have taken part in an audiorecorded consultation will be invited to take part in an interview; others who have not taken part in an audiorecorded consultation may also be invited to meet sampling requirements.

\section{Interview consent process}

Patients and healthcare professionals considered suitable for inclusion in the interviews (and any relatives/carers who will be present) will be provided with a participant information sheet.

Those willing to be approached by a researcher to take part in an interview will be asked to complete a consent to contact form, which will be securely electronically transferred to the qualitative researcher. The qualitative researcher will contact the participant, and if they are able and willing to take part, an interview will be arranged at a mutually convenient time. Consent will be taken verbally at the start of each interview and audio-recorded.

\section{Analysis}

\section{Analysis of audio-recorded consultations}

Audio-recorded consultations will be professionally transcribed verbatim. Discourse analysis will be used to examine shared decision-making and participants' talk around risk and uncertainty (particularly in relation to treatment options). Discourse analysis provides a rigorous, systematic approach to analysing naturally occurring interactions, exploring not only what is said but also how it is said. ${ }^{23}$ Theme-oriented discourse anal$\mathrm{ysis}^{24}$ will be conducted to explore detailed features of the interaction, such as intonation, vocabulary and pauses, drawing out key themes in relation to the research question. This analytic focus on interaction is particularly well suited to exploring complex discussions between healthcare professionals and patients. ${ }^{25}$

\section{Analysis of interviews}

Interviews will be professionally transcribed verbatim. Thematic analysis ${ }^{26}$ will be used to identify key patterns in the data. This will consist of a series of steps: familiarisation with data, generating initial codes and searching, reviewing and defining themes. Themes will be identified that relate to the objectives of the research, but analysis will also allow new, unpredicted themes generated by interviewees themselves to be identified. The analysis will identify contradictory data as points of contrast as well as similarities. Identified themes will be combined with the analysis of the audio-recorded consultations, to provide a detailed account of the communication, consent and shared decision-making process in relation to MLLA.

\section{Synthesis of qualitative findings with quantitative results}

Qualitative findings will be synthesised with results of the parallel quantitative component being carried out as part of PERCEIVE. Following the initial analysis of the qualitative and quantitative data sets, two data synthesis workshops will be held. Workshop participants will include the study qualitative researcher, statistician and surgeon, together with patients and relatives/carers (workshop 1) and healthcare professionals (workshop 2), to bring different conceptual perspectives into the analysis. The initial findings of the qualitative and quantitative analysis will be presented, to allow new interpretations once combined. It is hoped that member checking/respondent validation can be carried out, through the inclusion of patients, relatives/carers and healthcare professionals who took part in the audio-recorded consultations and/ or interviews. Workshop participants will be engaged to propose the content and format of (an) intervention(s) to improve shared decision-making and risk perception/ communication around MLLA. Following the workshop, a logic model for the intervention(s) and its future evaluation will be formulated by the study team.

\section{Patient and public involvement}

Two patient and public involvement (PPI) representatives (one who has undergone MLLA, the other a relative of an amputee) have been involved in the project from the development stage, with their experiences of amputation decision-making directly informing the aims of the research. A discussion group was held to refine the research objectives, attended by 13 patients/relatives, with four who were unable to attend providing written or verbal feedback. Participants described varied experiences in relation to communication and risk discussion around MLLA. The importance of involving patients' 
relatives was emphasised, as was the value of discussions and decisions taking place outside the ward environment. Their experiences informed the study design and resulted in specialist physiotherapists being included as a participant group.

The study PPI representatives provided advice and guidance on the development of the research plan, including planned recruitment and data collection processes. They are members of the study management group and have subsequently given feedback on participant information materials and interview topic guides. The data synthesis process will involve workshops with patients, relatives and healthcare professionals, to ensure that their perspectives are fully included in the analysis. PPI representatives will be asked for feedback on dissemination plans and materials and involved in publicising study findings via local media and relevant charities.

\section{Ethics and dissemination \\ Ethics}

The study has been approved by Wales REC 7 (20/ WA/0351). Informed consent will be obtained from all participants. Participants will be given a participant information sheet (or this will be read to them over the telephone), with sufficient time to consider the study information before consenting to participate. Patients will only be approached if their clinical condition allows sufficient time to obtain informed consent, and if the clinical team considers that participation would be appropriate. All participant information will be kept confidential.

\section{Dissemination}

In addition to the final report required by the funder, study findings will be published in international peerreviewed journal(s) and presented at national and international scientific meetings. With the assistance of collaborators and PPI representatives, the study team will disseminate to a wide NHS and lay audience and promote uptake of the study findings into clinical care. This will include presentations at meetings and written executive summaries for key stakeholder groups such as secondary care trusts, Royal Colleges, amputation charities and other relevant patient groups. Early dissemination has included an article about the study in the Limbless Association membership magazine StepForward.

\section{Summary}

To the best of our knowledge, this study will be the first to explore in-depth the amputation decision-making process, via examination of patient-surgeon consultations and interviews with key stakeholders. Findings will provide valuable insights into the perspectives of patients and healthcare professionals and the extent of shared decision-making. This increased understanding will inform the development of an intervention to improve decision-making around MLLA. Getting amputation decision-making right has the potential to have a considerable effect on patient outcomes, including quality of life.

Author affiliations

${ }^{1}$ Centre for Trials Research, Cardiff University, Cardiff, UK

${ }^{2}$ Gwent Vascular Institute, Royal Gwent Hospital, Aneurin Bevan University Health

Board, Newport, UK

${ }^{3}$ Department of General Surgery, University Hospitals Bristol and Weston NHS

Foundation Trust, Bristol, UK

${ }^{4}$ Artifical Limb and Appliance Centre, Rookwood Hospital, Cardiff and Vale University Health Board, Cardiff, UK

${ }^{5}$ Vascular Department, Cardiff and Vale University Health Board, Cardiff, UK

${ }^{6}$ Department of Anaesthesia, North Bristol NHS Trust, Bristol, UK

${ }^{7}$ C/O INVOLVE, Health and Care Research Wales, Cardiff, UK

${ }^{8}$ Vascular Department, North Bristol NHS Trust, Bristol, UK

${ }^{9}$ PRIME Centre Wales, Division of Population Medicine, Cardiff University, Cardiff, UK

Twitter Katie Samuel @katie_samuel_

Contributors DCB conceived the research idea and led the development of the original grant application. SM, LB-H, BLG, C-AW, ET-J, RP, PP, DH, IM, PS, KS, SJ, $D C, C P T$ and $A E$ contributed to the acquisition of funding via development and critical review of the grant application. LB-H, SM, AE and DCB developed the methods for the qualitative study. As PPI representatives, SJ and DC provided advice and guidance on the development of study data collection processes. C-AW is responsible for management and coordination of the study and study governance, with oversight from ET-J. SM wrote the protocol and prepared the first draft of the manuscript. All authors provided critical review and final approval of the protocol and manuscript.

Funding This work is supported by the Research for Patient and Public Benefit (RfPPB) programme, Health and Care Research Wales, grant number RfPPB19-1642. The Centre for Trials Research receives funding from Health and Care Research Wales and Cancer Research UK.

Competing interests None declared.

Patient consent for publication Not applicable.

Provenance and peer review Not commissioned; externally peer reviewed.

Supplemental material This content has been supplied by the author(s). It has not been vetted by BMJ Publishing Group Limited (BMJ) and may not have been peer-reviewed. Any opinions or recommendations discussed are solely those of the author(s) and are not endorsed by BMJ. BMJ disclaims all liability and responsibility arising from any reliance placed on the content. Where the content includes any translated material, BMJ does not warrant the accuracy and reliability of the translations (including but not limited to local regulations, clinical guidelines, terminology, drug names and drug dosages), and is not responsible for any error and/or omissions arising from translation and adaptation or otherwise.

Open access This is an open access article distributed in accordance with the Creative Commons Attribution Non Commercial (CC BY-NC 4.0) license, which permits others to distribute, remix, adapt, build upon this work non-commercially, and license their derivative works on different terms, provided the original work is properly cited, appropriate credit is given, any changes made indicated, and the use is non-commercial. See: http://creativecommons.org/licenses/by-nc/4.0/.

\section{ORCID iDs}

Sarah Milosevic http://orcid.org/0000-0003-1973-8286

Brenig LIwyd Gwilym http://orcid.org/0000-0002-5403-8720

Cherry-Ann Waldron http://orcid.org/0000-0001-8465-2492

Philip Pallmann http://orcid.org/0000-0001-8274-9696

Adrian Edwards http://orcid.org/0000-0002-6228-4446

\section{REFERENCES}

1 Columbo JA, Davies L, Kang R, et al. Patient experience of recovery after major leg amputation for arterial disease. Vasc Endovascular Surg 2018;52:262-8.

2 van Netten JJ, Fortington LV, Hinchliffe RJ, et al. Early post-operative mortality after major lower limb amputation: a systematic review of population and regional based studies. Eur J Vasc Endovasc Surg 2016;51:248-57. 
3 Klaphake S, de Leur K, Mulder PG, et al. Mortality after major amputation in elderly patients with critical limb ischemia. Clin Interv Aging 2017;12:1985-92.

4 Ambler GK, Thomas-Jones E, Edwards AGK, et al. Prognostic risk modelling for patients undergoing major lower limb amputation: an analysis of the UK National vascular registry. Eur $J$ Vasc Endovasc Surg 2020;59:606-13.

5 Schrier E, Dijkstra PU, Zeebregts CJ, et al. Decision making process for amputation in case of therapy resistant complex regional pain syndrome type-I in a Dutch specialist centre. Med Hypotheses 2018;121:15-20.

6 Franklin H, Rajan M, Tseng C-L, et al. Cost of lower-limb amputation in U.S. veterans with diabetes using health services data in fiscal years 2004 and 2010. J Rehabil Res Dev 2014;51:1325-30.

7 Rassin M, Tzevlin V, Malul E, et al. Retrospective study of emerging themes in the decision-making process of patients considering amputation. J Vasc Nurs 2012;30:54-60.

8 Dilaver NM, Gwilym BL, Preece R, et al. Systematic review and narrative synthesis of surgeons' perception of postoperative outcomes and risk. BJS Open 2020;4:16-26.

9 Dillon MP, Quigley M, Fatone S. Outcomes of dysvascular partial foot amputation and how these compare to transtibial amputation: a systematic review for the development of shared decision-making resources. Syst Rev 2017;6:54.

10 Hoffmann TC, Légaré F, Simmons MB, et al. Shared decision making: what do clinicians need to know and why should they bother? Med $J$ Aust 2014;201:35-9.

11 Brothers TE, Robison JG, Elliott BM. Prospective decision analysis for peripheral vascular disease predicts future quality of life. J Vasc Surg 2007;46:701-8.

12 General Medical Council. Decision making and consent, 2020. Available: https://www.gmc-uk.org/ethical-guidance/ethicalguidance-for-doctors/decision-making-and-consent [Accessed 28 Jan 2021].

13 de Mik SML, Stubenrouch FE, Balm R, et al. Systematic review of shared decision-making in surgery. Br J Surg 2018;105:1721-30.

14 Joseph-Williams N, Williams D, Wood F, et al. A descriptive model of shared decision making derived from routine implementation in clinical practice ('Implement-SDM'): Qualitative study. Patient Educ Couns 2019;102:1774-85.
15 Vascular Society of Great Britain and Ireland. Vascular Society of great britain and ireland 2018 Yearbook, 2018. Available: https:// www.vascularsociety.org.uk/_userfiles/pages/files/Annual\% 20Meeting/2018ASM/Yearbook\%202018.pdf [Accessed 29 Jan 2021]

16 Conte MS, Bradbury AW, Kolh P, et al. Global vascular guidelines on the management of chronic limb-threatening ischemia. Eur $J$ Vasc Endovasc Surg 2019;58:e33:S1-109.

17 Connelly J, Airey M, Chell S. Variation in clinical decision making is a partial explanation for geographical variation in lower extremity amputation rates. Br J Surg 2001;88:529-35.

18 Gwilym BL, Waldron C-A, Thomas-Jones E, et al. The perceive quantitative study: prediction of risk and communication of outcome following major lower-limb amputation: protocol for a collaboratiVE study. BJS Open 2021;5. doi:10.1093/bjsopen/zrab118. [Epub ahead of print: 09 Nov 2021].

19 Malterud K, Siersma VD, Guassora AD. Sample size in qualitative interview studies: guided by information power. Qual Health Res 2016;26:1753-60.

20 Bernat JL, Peterson LM. Patient-Centered informed consent in surgical practice. Arch Surg 2006;141:86-92.

21 Edwards A, Elwyn G, Hood K, et al. The development of COMRADE-a patient-based outcome measure to evaluate the effectiveness of risk communication and treatment decision making in consultations. Patient Educ Couns 2003;50:311-22.

22 Elwyn G, Tsulukidze M, Edwards A, et al. Using a 'talk' mode of shared decision making to propose an observation-based measure: Observer OPTION 5 Item. Patient Educ Couns 2013;93:265-71.

23 Traynor M. Discourse analysis: theoretical and historical overview and review of papers in the Journal of advanced nursing 1996-2004. $J$ Adv Nurs 2006;54:62-72.

24 Roberts C, Sarangi S. Theme-oriented discourse analysis of medical encounters. Med Educ 2005;39:632-40.

25 Sherlock R, Wood F, Joseph-Williams N, et al. "What would you recommend doctor?"-Discourse analysis of a moment of dissonance when sharing decisions in clinical consultations. Health Expect 2019;22:547-54

26 Braun V, Clarke V. Using thematic analysis in psychology. Qual Res Psychol 2006;3:77-101. 